\title{
Interactive comment on "AMOC-emulator M-AMOC1.0 for uncertainty assessment of future projections" by Pepijn Bakker and Andreas Schmittner
}

\author{
P. G. Myers \\ pmyers@ualberta.ca \\ Received and published: 9 June 2016
}

This paper develops an AMOC emulator to mimic the results of a small ensemble of runs from a given climate model. After discussing the method and showing how the emulator can represent the main aspects of key variables in the ensemble, the authors show how their simple tool can then be used to examine additional aspects of the parameter space that might be hard to cover with a more computationally expensive climate model. They also evaluate their approach using additional runs used for the training ensemble.

This is a well written and clear paper that is easy to follow. The need for such a tool is well justified. Figures are clear. Evaluation for the case presented is strong. Thus, 
I think this paper is appropriate for the literature, and this journal. I would recommend acceptance with only a small number of minor revisions.

page 2, Line 18: Would the approach work in a GCM whose AMOC is controlled by the strength of southern hemisphere winds? This is a potential major caveat, that might be worth discussing more at the end of the paper when the authors expand on applications.

page 4, line 12: Is it reasonable to assume the wind-driven oceanic meridional freshwater transport is static. I might expect it to evolve with an evolving climate, especially as high latitude freshwater sources (e.g. GIS runoff) changes.

Page 5, line 30: algorithm misspelled.

page 6, line 4: Why fixed limits of plus/minus $200 \%$ ? Why not something based on each variable's variability/range?

page 6 , line 8 - analogous misspelled.

Summary: Way too short. I'd like to see more discussion on how such an emulator could be improved, its limitations, questions it might be best for, etc.

Table 1: For last 3 entries, dependent misspelled

Figure 1: Why is the atmospheric part squished so much in the vertical compared to the oceanic component?

Figure 4 caption: relative misspelled.

Figure 8 caption: right misspelled.

Interactive comment on Geosci. Model Dev. Discuss., doi:10.5194/gmd-2016-79, 2016.

Printer-friendly version

Discussion paper 